\title{
Percutaneous Microwave Ablation versus Partial Splenic Embolization for Treatment of Hypersplenism in Patients with Liver Cirrhosis
}

\author{
Yousef Mohammed Asar ${ }^{1}$, Mostafa Soliman El Kady ${ }^{1}$, \\ Hatem Samir Alegaily ${ }^{1}$, Waleed Ahmed El Agawy ${ }^{2}$, \\ Tamer El Eraki ${ }^{1}$, Wessam Mostafa Abd El Latif ${ }^{3}$ \\ ${ }^{1}$ Hepatology, Gastroentrology and Infectious Diseases Department, Faculty of Medicine, Benha \\ University, Egypt \\ ${ }^{2}$ Tropical Medicine Department, Faculty of Medicine, Port Said University, Port Said, Egypt \\ ${ }^{3}$ Fellow of Diagnostic and Interventional Radiology, National Hepatology and Tropical Medicine \\ Institute, Cairo, Egypt
}

Corresponding Author Asar, Yousef Mohammed

Mobile:

$+201098807883$

E mail:

yousefasar815@gmail. com

Key words: Liver cirrhosis, Hypersplenism, Percutaneous microwave ablation, Partial splenic embolization
Background and study aim: In patients suffering from portal hypertension as result of liver cirrhosis, hypersplenism was ranged from 11 to $55 \%$. The aim of our work was to compare between percutaneous microwave ablation (MWA) and partial splenic artery embolization (PSE) in the management of hypersplenism in patients with liver cirrhosis as regard efficacy and safety.

Patients and Methods: Sixty patients with liver cirrhosis complicated with splenomegaly and hypersplenism were divided randomly into three groups; Group (1): Twenty patients were underwent one session of MW ablation of splenic parenchyma with target ablation volume about 20\%, Group (2): Twenty patients were underwent two sessions of MW ablation of splenic parenchyma with 1 week interval with target ablation up to $40 \%$, Group (3): Twenty patients were underwent PSE.

Results: There was an improvement in the hemoglobin, platelet and leucocyte levels in three groups, which was $9.47 \pm$ $2.14 \mathrm{gm} / \mathrm{dl}, 42.75 \pm 15.4 \times 10^{3} / \mathrm{mm}^{3}$ and $2.6 \pm 0.5 \times 10^{3} / \mathrm{mm}^{3}$ before the procedure respectively and become $9.95 \pm 2.29$

\section{INTRODUCTION}

Hypersplenism is a syndrome in which there is enlargement of the spleen, associated with a decrease in one or more of the cell lines of the peripheral blood with normocellular or hypercellular bone marrow and this deficit is corrected by splenectomy.

Asar et al., Afro-Egypt J Infect Endem Dis 2019; 9(3):230-240

https://aeji.journals.ekb.eg/

http://mis.zu.edu.eg/ajied/home.aspx $\mathrm{gm} / \mathrm{dl}, 313.5 \pm 99.6 \times 10^{3} / \mathrm{mm}^{3}$ and $6.88 \pm$ $1.89 \times 10^{3} / \mathrm{mm}^{3}$ after one month as regard PSE group. As regard one session of MWA was $9.73 \pm 2.02 \mathrm{gm} / \mathrm{dl}, 45.57 \pm 11.2$ $\mathrm{x} 10^{3} / \mathrm{mm}^{3}$ and $2.8 \pm 0.85 \times 10^{3} / \mathrm{mm}^{3}$ and become $11.83 \pm 0.74 \mathrm{gm} / \mathrm{dl}, \quad 152 \pm 26.43$ $\mathrm{x} 10^{3} / \mathrm{mm}^{3}$ and $5.85 \pm 1.01 \times 10^{3} / \mathrm{mm}^{3}$ after one month. As regard two sessions of MWA group; was $9.2 \pm 2.15 \mathrm{gm} / \mathrm{dl}, 40.6 \pm$ $11.5 \times 10^{3} / \mathrm{mm}^{3}$ and $2.4 \pm 0.55 \times 10^{3} / \mathrm{mm}^{3}$ and become $12.74 \pm 1.2 \mathrm{gm} / \mathrm{dl}, 183.4 \pm$ $26.43 \times 10^{3} / \mathrm{mm}^{3}$ and $6.29 \pm 1.17 \times 10^{3} / \mathrm{mm}^{3}$ after one month. PSE was significantly more effective in the elevation of platelets and leucocytes than other two groups $(\mathrm{p}=0.00)$, and two sessions of MWA more than one session of MWA. The two sessions MWA group was significantly higher than other two groups while one session of MWA group was more than PSE group in the elevation of hemoglobin $(p=0.00)$. No mortality occurred in the three groups, but PSE was associated with more serious complications than the other two groups.

Conclusion: MWA and PSE were effective in treatment of hypersplenism in patients with liver cirrhosis, while percutaneous MWA has less serious complications than PSE.

However, there are cases such as essential thrombocytopenic purpura where the spleen may be not enlarged [1].

When the underlying disease causing hypersplenism cannot be determined, it is called primary hypersplenism [2]. 
When the underlying cause of hypersplenism can be identified, it is called secondary hypersplenism. The most common cause of secondary hypersplenism is the chronic liver disease [3].

The cause of the liver disease influences the frequency of splenomegaly. Patients with idiopathic portal hypertension typically have large spleens, and it has been reported that patients with non-alcoholic cirrhosis tend to have larger spleens than patients with alcoholic cirrhosis [4].

Hypersplenism associated with retention of a large number of leukocytes, erythrocytes and platelets in the spleen. The number of retained blood cells can be 5.5-20 times higher than the normal level, thus facilitating capture, phagocytosis or destruction of blood cells by phagocytes resulting in peripheral cytopenia [5].

Due to the low platelets count , patients develop bleeding manifestations of skin and mucous membranes and reduction in red blood cells causing anemia which in turn leads to lack of oxygen and disturbance of daily activities as well as neutropenia lead to increased vulnerability for the infections which may be very severe and fatal. So hypersplenism with recurrent spontaneous bleeding, frequent blood transfusion or severe recurrent infection is a clinical indication for therapeutic intervention [6].

However, this blood cell destruction can be eliminated by splenectomy, but the incidence of severe complications after splenectomy is depending on whether laparoscopic or open splenectomy is achieved [7].

In 1973, The first splenic parenchymal embolization was achieved by Maddison [8].

However, PSE was effective but associated with many adverse events, like fever, vomiting, abdominal pain, splenic rupture, splenic abscess, pneumonia, deterioration of ascites, pleural effusion and hematemesis [9].

Minimally invasive procedures like thermal ablation methods using different energy sources, like microwave (MW), radiofrequency (RF), or laser, were developed for the elimination of local tumor tissue within solid organs [10].

Either percutaneous or laparoscopic microwave ablation is safe approach for the management of hypersplenism as a consequence of cirrhosis of liver [11].

\section{PATIENTS AND METHODS}

In this prospective randomized study sixty patients with post-HCV liver cirrhosis complicated with splenomegaly and hypersplenism attended to Egyptian National Hepatology Institute in Cairo in the period from January 2017 to March 2019 with clinically severe hypersplenism. They were randomly allocated into three groups: Group (1) Twenty patients were treated with one session of microwave ablation of parenchyma of spleen with target ablation volume about $20 \%$, Group (2): Twenty patients were treated with two sessions of microwave ablation of parenchyma of spleen with one-week interval between two sessions with target ablation about 40\% and Group (3) Twenty patients were treated with Partial splenic embolization.

All patients were subjected to full history taking, thorough clinical examination and detailed investigations (bilirubin level, serum albumin, renal functions, INR and full blood count). Diagnosis was based on full blood count and confirmed with bone marrow examination and diagnosis of portal hypertension and liver cirrhosis was through clinical examination and laboratory and radiological investigations.

Patients with hypocellular bone marrow, significant cardiac disease, renal impairment, malignant disease, child class $\mathrm{C}$, those with a known allergy to contrast medium and other causes of cytopenia, which may include autoimmune destruction; deficiencies of iron, vitamin B12, or folate; were excluded from the study.

Procedure for splenic microwave ablation: Administration of propofol by anesthetist in operating theatre under monitoring. abdominal wall sterilization, then the microwave needle (AMICA $^{\mathrm{TM}}$ - MW Ablation System) was inserted in the splenic parenchyma under ultrasound guidance beyond large vessels and surrounding organs by expert hepatologist.ECO-100C microwave treatment system Nanjing ECO Microwave System Engineering Co., Ltd. Italy. With the MW emitting 80 Watt for 10 minutes was used in the ablation in one session to achieve volume ablation about 20\% as done by Assal et al. [12]. When ablation was completed needle track ablation was achieved to avoid post procedural bleeding.

Procedure for partial splenic embolization: through femoral artery approach splenic artery catheterization using the introducer sheath, dilator and guide wire was done according to 
N'Kontchou et al. [24] by expert interventional radiologist. Then the embolizing agent (Embosphere) mixed with lipiodol was injected through special catheters in small increments, the injection of one or two of the polar arteries (according to their size) was sufficient to embolize at least $40-60 \%$ of the splenic parenchyma after the embolization was complete, splenic angiography was done to confirm a reduction of approximately $40-60 \%$ of the splenic blood flow.

All patients stayed in hospital after procedure under follow up. Supported care included fluid intake, prophylaxis with IV Cefotaxime $1 \mathrm{gm}$ before procedure and continued every 12 hours for five days and Paracetamol 500mg three times daily for 1 week. All patients in three groups were followed up after discharge at 1 week, 1 month, and 3 months.

\section{Ethical approved:}

Our work was approved by the research ethical committee of Benha faculty of medicine. All included patients were informed about the idea of the study and gave a written consent to participate in the study.

\section{Statistical analysis:}

Patient data were statistically analyzed using SPSS. Qualitative data were expressed as number and percentage. Quantitative data were expressed as mean \pm standard deviation (SD). The following tests were used to test differences for significance; Difference and association of qualitative variable by Chi square test $\left(\mathrm{X}^{2}\right)$. Differences between quantitative independent multiple by ANOVA or Kruskal Wallis, paired by paired t or Sign. And post hoc analysis was used to know what specific pairs of group means show differences and what pairs do not. $\mathrm{P}$ value was set at $<0.05$ for significant results $\&<0.001$ for high significant results.

\section{RESULTS}

This prospective randomized study was conducted on 60 patients with hypersplenism secondary to cirrhosis, they were 33 (55\%) males and 27 (45\%) females. One session MWA group: composed of 10 males (50\%) and 10 Females (50\%). Two sessions MWA group: composed of 10 males $(50 \%)$ and 10 Females (50\%). PSE group: composed of 13 males $(65 \%)$ and 7 Females
(35\%). No significant statistical difference was detected between the three groups as regarding gender distribution $(\mathrm{p}=0.5)$ (Table 1).

The age was distributed as $41.9 \pm 10.33$ years in PSE group, 42.05 \pm 7.87 years in one session MWA and $46.7 \pm 9.12$ years in two sessions MWA group with no statistically significant difference between them $(\mathrm{p}=0.17)$ (Table1).

Splenic size before the intervention was distributed as $20.5 \pm 2.07 \mathrm{~cm}$ in PSE group, $20.2 \pm 1.67 \mathrm{~cm}$ in one session MWA and $20.4 \pm 1.21 \mathrm{~cm}$ in two sessions MWA group with no statistically significant difference among the studied groups $(\mathrm{p}=0.17)$ (Table 1).

The laboratory finding before the intervention including, hemoglobin level, leucocytic and platelets counts, INR, bilirubin level, albumin and creatinine level show no statistically significant difference among the three groups (Table 2).

The laboratory findings after the interventions at different intervals show: Hemoglobin showed significant difference among groups start from the first month $(9.95 \pm 2.29 \mathrm{gm} / \mathrm{dl}$ for PSE, $11.83 \pm$ $0.74 \mathrm{gm} / \mathrm{dl}$ for one session MWA and 12.74 \pm 1.2 $\mathrm{gm} / \mathrm{dl}$ for two session MWA) $(\mathrm{p}=0.01)$ and continue after 3 months $(\mathrm{p}=0.002)$ as two sessions MWA group $(12.35 \pm 1.29 \mathrm{gm} / \mathrm{dl})$ significantly higher than other two groups and one session MWA group significantly higher than PSE group (Table 3 \& Table 4). Leucocyte count; show significant difference among groups start from the first week $\left(7.35 \pm 1.1 \times 10^{3} / \mathrm{mm}^{3}\right.$ for PSE, $4.73 \pm 1.07 \times 10^{3} / \mathrm{mm}^{3}$ for one session MWA and $5.05 \pm 1.09 \times 10^{3} / \mathrm{mm}^{3}$ for two session MWA) $(\mathrm{p}=0.003)$ as PSE group significantly higher than other groups then from first month two sessions MWA group become significantly higher than one session MWA group (Table $3 \&$ Table 5). Platelets count; show significant difference from1week till the end as PSE group significantly higher than other two groups $(\mathrm{p}=0.00)$ and two sessions group more than one session significantly from first month $\left(252.75 \pm 60.69 \times 10^{3} / \mathrm{mm}^{3}\right.$ for PSE, $126.95 \pm 27.43$ $\mathrm{x} 10^{3} / \mathrm{mm}^{3}$ for one session MWA and $155.95 \pm 26.43 \times 10^{3} / \mathrm{mm}^{3}$ for two session MWA after three months) (Table $3 \&$ Table 6).

Albumin, bilirubin and INR; show that from first week till the end albumin was significantly lower in PSE group than other two groups but bilirubin was significantly higher in PSE group from one 
week tell the end $(\mathrm{p}=0.00)$ and INR at third month PSE significantly higher than other groups $(p=0.019) \quad$ (Table 3). Creatinine; shows no significant difference among groups after the intervention (Table 3).

There is no significance difference among the three studied groups in ascites state before the intervention $(\mathrm{p}=0.7)$ and PSE group significantly associated with deterioration of the ascites state after 3 months from the intervention $(\mathrm{p}=0.002)$ (Table 7).

PSE group significantly associated with deterioration in child Turcotte Pugh score $(\mathrm{p}=0.00)$ while no change after the intervention in other two groups (Table 8).

The post-intervention complications; revealed that one patient suffered from severe left upper abdominal pain after one session of microwave.
While two patients suffered from severe left upper abdominal pain and two patients developed a small amount of pleural effusion after two sessions of microwave. In PSE group six patients developed partial portal vein thrombosis due to rapid rise of platelets level after the intervention which was managed by anticoagulant, three patients developed pleural effusion, two patients developed hematemesis which related to PSE because we excluded patients with active esophageal varices from our study, two patients developed paralytic ileus and nine patients suffered from left upper abdominal pain so PSE group associated with more serious complications and there were no patients had developed splenic abscess or sepsis after the intervention (Table 9).

Table (1): Demographic and base line data of the studied groups.

\begin{tabular}{|c|c|c|c|c|c|c|}
\hline & & \multicolumn{3}{|c|}{ Groups } & \multirow[b]{2}{*}{$\mathbf{X}^{2} / \mathrm{F}$} & \multirow[b]{2}{*}{$\mathbf{P}$} \\
\hline & & PSE group & $\begin{array}{l}\text { One session of } \\
\text { MWA group }\end{array}$ & $\begin{array}{c}\text { Two sessions of } \\
\text { MWA group }\end{array}$ & & \\
\hline \multirow{4}{*}{ Sex } & Tol & 13 & 10 & 10 & \multirow{4}{*}{1.21} & \multirow{4}{*}{0.54} \\
\hline & ivale & $65.0 \%$ & $50.0 \%$ & $50.0 \%$ & & \\
\hline & \multirow{2}{*}{ Female } & 7 & 10 & 10 & & \\
\hline & & $35.0 \%$ & $50.0 \%$ & $50.0 \%$ & & \\
\hline \multicolumn{2}{|c|}{$\begin{array}{l}\text { Age (years) } \\
\text { Mean } \pm \text { SD }\end{array}$} & $41.9 \pm 10.33$ & $42.05 \pm 7.87$ & $46.7 \pm 9.12$ & 1.774 & 0.17 \\
\hline \multicolumn{2}{|c|}{$\begin{array}{c}\text { Spleen }(\mathrm{cm}) \\
\text { Mean } \pm \text { SD } \\
\text { Range }\end{array}$} & $\begin{array}{c}20.5 \pm 2.07 \\
17-25\end{array}$ & $\begin{array}{c}20.2 \pm 1.67 \\
16-22\end{array}$ & $\begin{array}{c}20.4 \pm 1.21 \\
17-22\end{array}$ & 1.765 & 0.17 \\
\hline
\end{tabular}

(PSE: partial splenic embolization, MWA: microwave ablation, X2: Chi-square, F: ANNOVA test, $p<0.05$ is significant).

Table (2): Laboratory finding before intervention among studied groups.

\begin{tabular}{|c|c|c|c|c|c|}
\hline Dependent Variable & PSE group & $\begin{array}{l}\text { One session } \\
\text { MWA group }\end{array}$ & $\begin{array}{l}\text { Two session } \\
\text { MWA group }\end{array}$ & $\mathbf{F}$ & $\mathbf{P}$ \\
\hline Platelets $\left(1000 / \mathrm{mm}^{3}\right)$ & $42.75 \pm 15.4$ & $45.75 \pm 11.2$ & $40.6 \pm 11.5$ & 0.8 & 0.45 \\
\hline Hemoglobin (gm/dl) & $9.47 \pm 2.14$ & $9.37 \pm 2.02$ & $9.2 \pm 2.15$ & 1.033 & 0.4 \\
\hline WBCs $\left(1000 / \mathrm{mm}^{3}\right)$ & $2.6 \pm 0.5$ & $2.8 \pm 0.58$ & $2.4 \pm 0.55$ & 2.5 & 0.29 \\
\hline Albumin (gm/dl) & $3.38 \pm 0.36$ & $3.42 \pm 0.51$ & $3.61 \pm 0.52$ & 1.609 & 0.21 \\
\hline Bilirubin (mg/dl) & $1.45 \pm 0.47$ & $1.18 \pm 0.49$ & $1.41 \pm 0.53$ & 1.655 & 0.2 \\
\hline INR & $1.18 \pm 0.2$ & $1.23 \pm 0.21$ & $1.25 \pm 0.21$ & 0.49 & 0.61 \\
\hline Creatinine (mg/dl) & $1.25 \pm 0.21$ & $0.98 \pm 0.21$ & $0.99 \pm 0.2$ & 1.960 & 0.1 \\
\hline
\end{tabular}

( $\mathrm{F}=$ ANOVA test, WBCs: white blood cells, INR: international normalized ratio, PSE: partial splenic embolization, MWA: microwave ablation) 
Table (3): Laboratory finding after the intervention among studied groups.

\begin{tabular}{|c|c|c|c|c|c|c|}
\hline \multicolumn{2}{|c|}{ Dependent Variable } & PSE group & $\begin{array}{l}\text { One session } \\
\text { MWA group }\end{array}$ & $\begin{array}{c}\text { Two session } \\
\text { MWA group }\end{array}$ & $\mathbf{F}$ & $\mathbf{P}$ \\
\hline \multirow{3}{*}{$\begin{array}{c}\text { Platelets } \\
\left(1000 / \mathbf{m m}^{3}\right)\end{array}$} & $\begin{array}{c}\text { After one } \\
\text { week }\end{array}$ & $340.55 \pm 77.5$ & $110.0 \pm 42.5$ & $111.15 \pm 30.5$ & 20.716 & $0.00 *$ \\
\hline & $\begin{array}{c}\text { After } 1 \\
\text { month }\end{array}$ & $313.5 \pm 99.6$ & $152.0 \pm 26.43$ & $183.4 \pm 26.43$ & 89.076 & $0.00 *$ \\
\hline & $\begin{array}{l}\text { After } 3 \\
\text { months }\end{array}$ & $252.75 \pm 60.69$ & $126.95 \pm 27.43$ & $155.95 \pm 26.43$ & 49.052 & $0.00^{*}$ \\
\hline \multirow{3}{*}{$\begin{array}{l}\text { Hemoglobin } \\
\text { (gm/dl) }\end{array}$} & $\begin{array}{c}\text { After one } \\
\text { week }\end{array}$ & $9.75 \pm 0.84$ & $9.61 \pm 1.48$ & $9.7 \pm 2.57$ & 0.6 & 0.509 \\
\hline & $\begin{array}{c}\text { After } 1 \\
\text { month }\end{array}$ & $9.95 \pm 2.29$ & $11.83 \pm 0.74$ & $12.74 \pm 1.2$ & 4.62 & $0.01 *$ \\
\hline & $\begin{array}{l}\text { After } 3 \\
\text { months }\end{array}$ & $10.88 \pm 2.35$ & $11.76 \pm 1.1$ & $12.35 \pm 1.29$ & 5.304 & $0.002 *$ \\
\hline \multirow{3}{*}{$\begin{array}{c}\text { WBCs } \\
\left(1000 / \mathrm{mm}^{3}\right)\end{array}$} & $\begin{array}{c}\text { After one } \\
\text { week }\end{array}$ & $7.35 \pm 1.1$ & $4.73 \pm 1.07$ & $5.05 \pm 1.09$ & 4.533 & $0.003 *$ \\
\hline & $\begin{array}{c}\text { After } 1 \\
\text { month }\end{array}$ & $6.88 \pm 1.89$ & $5.85 \pm 1.01$ & $6.29 \pm 1.17$ & 5.228 & $0.001 * *$ \\
\hline & $\begin{array}{l}\text { After } 3 \\
\text { months }\end{array}$ & $6.36 \pm 1.78$ & $5.15 \pm 1.31$ & $6.18 \pm 1.27$ & 4.865 & $0.002 *$ \\
\hline \multirow{3}{*}{$\begin{array}{l}\text { Albumin } \\
\text { (gm/dl) }\end{array}$} & $\begin{array}{c}\text { After one } \\
\text { week }\end{array}$ & $3.11 \pm 0.31$ & $3.58 \pm 0.69$ & $3.69 \pm 0.53$ & 4.663 & $0.013^{*}$ \\
\hline & $\begin{array}{c}\text { After } 1 \\
\text { month }\end{array}$ & $3.02 \pm 0.28$ & $3.45 \pm 0.6$ & $3.68 \pm 0.57$ & 6.75 & $0.002 *$ \\
\hline & $\begin{array}{l}\text { After } 3 \\
\text { months }\end{array}$ & $2.98 \pm 0.3$ & $3.44 \pm 0.61$ & $3.62 \pm 0.57$ & 7.944 & $0.001 * *$ \\
\hline \multirow{3}{*}{$\begin{array}{l}\text { Bilirubin } \\
\text { (mg/dl) }\end{array}$} & $\begin{array}{c}\text { After one } \\
\text { week }\end{array}$ & $3.29 \pm 1.21$ & $1.26 \pm 0.5$ & $1.56 \pm 0.8$ & 12.323 & $0.00^{* *}$ \\
\hline & $\begin{array}{c}\text { After } 1 \\
\text { month }\end{array}$ & $5.48 \pm 1.87$ & $1.46 \pm 0.66$ & $1.57 \pm 0.54$ & 61.453 & $0.00 * *$ \\
\hline & $\begin{array}{l}\text { After } 3 \\
\text { months }\end{array}$ & $4.52 \pm 2.12$ & $1.51 \pm 0.65$ & $1.78 \pm 0.54$ & 10.555 & $0.00 * *$ \\
\hline \multirow{3}{*}{ INR } & $\begin{array}{c}\text { After one } \\
\text { week }\end{array}$ & $1.22 \pm 0.2$ & $1.24 \pm 0.2$ & $1.27 \pm 0.18$ & 0.391 & 0.391 \\
\hline & $\begin{array}{l}\text { After } 1 \\
\text { month } \\
\end{array}$ & $1.32 \pm 0.2$ & $1.27 \pm 0.21$ & $1.28 \pm 0.21$ & 0.470 & 0.6 \\
\hline & $\begin{array}{l}\text { After } 3 \\
\text { months }\end{array}$ & $1.54 \pm 0.26$ & $1.3 \pm 0.22$ & $1.31 \pm 0.2$ & 4.201 & $0.019 *$ \\
\hline \multirow{3}{*}{$\begin{array}{l}\text { Creatinine } \\
\text { (mg/dl) }\end{array}$} & $\begin{array}{c}\text { After one } \\
\text { week }\end{array}$ & $1.29 \pm 0.21$ & $1.19 \pm 0.22$ & $1.17 \pm 0.2$ & 1.847 & 0.162 \\
\hline & $\begin{array}{c}\text { After } 1 \\
\text { month } \\
\end{array}$ & $1.46 \pm 0.31$ & $1.51 \pm 1.77$ & $1.0 \pm 0.17$ & 1.427 & 0.24 \\
\hline & $\begin{array}{l}\text { After } 3 \\
\text { months }\end{array}$ & $1.52 \pm 0.26$ & $1.06 \pm 0.16$ & $1.46 \pm 1.78$ & 1.118 & 0.33 \\
\hline
\end{tabular}

There is significant difference between studied groups. ( $\mathrm{F}=$ ANOVA test, WBCs: white blood cells, INR: international normalized ratio, PSE: partial splenic embolization, MWA: microwave ablation) 
Table (4): Post hoc analysis of hemoglobin difference among studied groups

\begin{tabular}{|c|c|c|c|}
\hline Dependent variable & Group & Group & P \\
\hline \multirow{3}{*}{ HB-1 month } & PSE group & One session of MWA group & .000 \\
\cline { 2 - 4 } & & Two sessions of MWA group & .000 \\
\cline { 2 - 4 } & One session of MWA & PSE group & .000 \\
\cline { 2 - 4 } & group & Two sessions of MWA group & .020 \\
\hline \multirow{2}{*}{ HB-3 month } & PSE group & One session of MWA group & .000 \\
\cline { 2 - 4 } & & Two sessions of MWA group & .000 \\
\cline { 2 - 4 } & $\begin{array}{c}\text { One session of MWA } \\
\text { group }\end{array}$ & PSE group & .000 \\
\cline { 2 - 4 } & & Two sessions of MWA group & .020 \\
\hline
\end{tabular}

There is significant difference among groups start from first month and continue after 3 months as two sessions group significantly higher than other two groups and one session group significantly higher than PSE group. (HB: hemoglobin, PSE: partial splenic embolization, MWA: microwave ablation).

Table (5): Post hoc analysis of leukocyte count difference among studied groups.

\begin{tabular}{|c|c|c|c|}
\hline Dependent Variable & Group & Group & P \\
\hline WBCs-1 week & PSE group & One session of MWA group & .000 \\
\cline { 2 - 4 } & & Two sessions of MWA group & .000 \\
\cline { 2 - 4 } & One session of MWA & PSE group & .000 \\
\cline { 2 - 4 } & group & Two sessions of MWA group & .725 \\
\hline \multirow{2}{*}{ WBCs -1 month } & PSE group & One session of MWA group & .000 \\
\cline { 2 - 4 } & & Two sessions of MWA group & .000 \\
\cline { 2 - 4 } & One session of MWA & PSE group & .000 \\
\cline { 2 - 4 } & group & Two sessions of MWA group & .044 \\
\cline { 2 - 4 } & PSE group & One session of MWA group & .000 \\
\cline { 2 - 4 } & & Two sessions of MWA group & .000 \\
\cline { 2 - 4 } & One session of MWA & PSE group & .000 \\
\cline { 2 - 4 } & group & Two sessions of MWA group & .035 \\
\hline
\end{tabular}

There is significant difference among groups Start from first 1st week as PSE group significantly higher than other groups then from 1st month two sessions group become significantly higher than one session. (WBCs: white blood cells, PSE: partial splenic embolization, MWA: microwave ablation)

Table (6): post hoc analysis of platelets count difference among studied groups.

\begin{tabular}{|c|c|c|c|}
\hline Dependent Variable & Group & Group & $\mathbf{P}$ \\
\hline \multirow[t]{4}{*}{ Platelets-1 week } & \multirow[t]{2}{*}{ PSE group } & One session of MWA group & .000 \\
\hline & & Two sessions of MWA group & .000 \\
\hline & \multirow[t]{2}{*}{ One session group } & PSE group & .000 \\
\hline & & Two sessions of MWA group & .725 \\
\hline \multirow[t]{4}{*}{ Platelets-1 month } & \multirow[t]{2}{*}{ PSE group } & One session of MWA group & .000 \\
\hline & & Two sessions of MWA group & .000 \\
\hline & \multirow[t]{2}{*}{ One session group } & PSE group & .000 \\
\hline & & Two sessions of MWA group & .000 \\
\hline \multirow[t]{4}{*}{ Platelets_3MONTH } & \multirow[t]{2}{*}{ PSE group } & One session of MWA group & .000 \\
\hline & & Two sessions of MWA group & .000 \\
\hline & \multirow[t]{2}{*}{ One session group } & PSE group & .000 \\
\hline & & Two sessions of MWA group & .000 \\
\hline
\end{tabular}

PSE group significantly higher than other two groups from one week till the end and two sessions group more than one session significantly from 1st month. (PSE: partial splenic embolization, MWA: microwave ablation) 
Table (7): Distribution of ascites among groups before and after intervention.

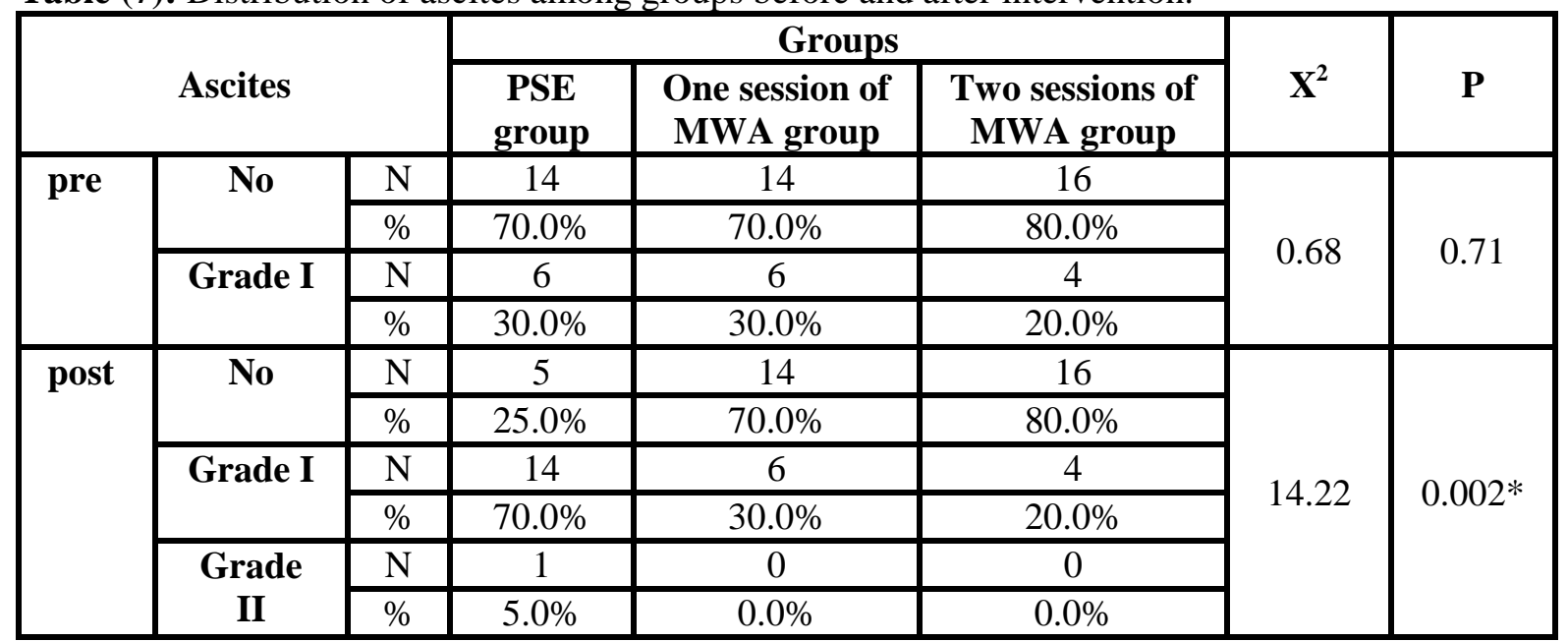

There is deterioration in ascites in PSE group after 3 months $(\mathrm{P}=0.002)$ while no changes in other two groups. (PSE: partial splenic embolization, MWA: microwave ablation, X2: Chi-square)

Table (8): Child Turcotte Pugh score of patients before and after intervention in studied groups.

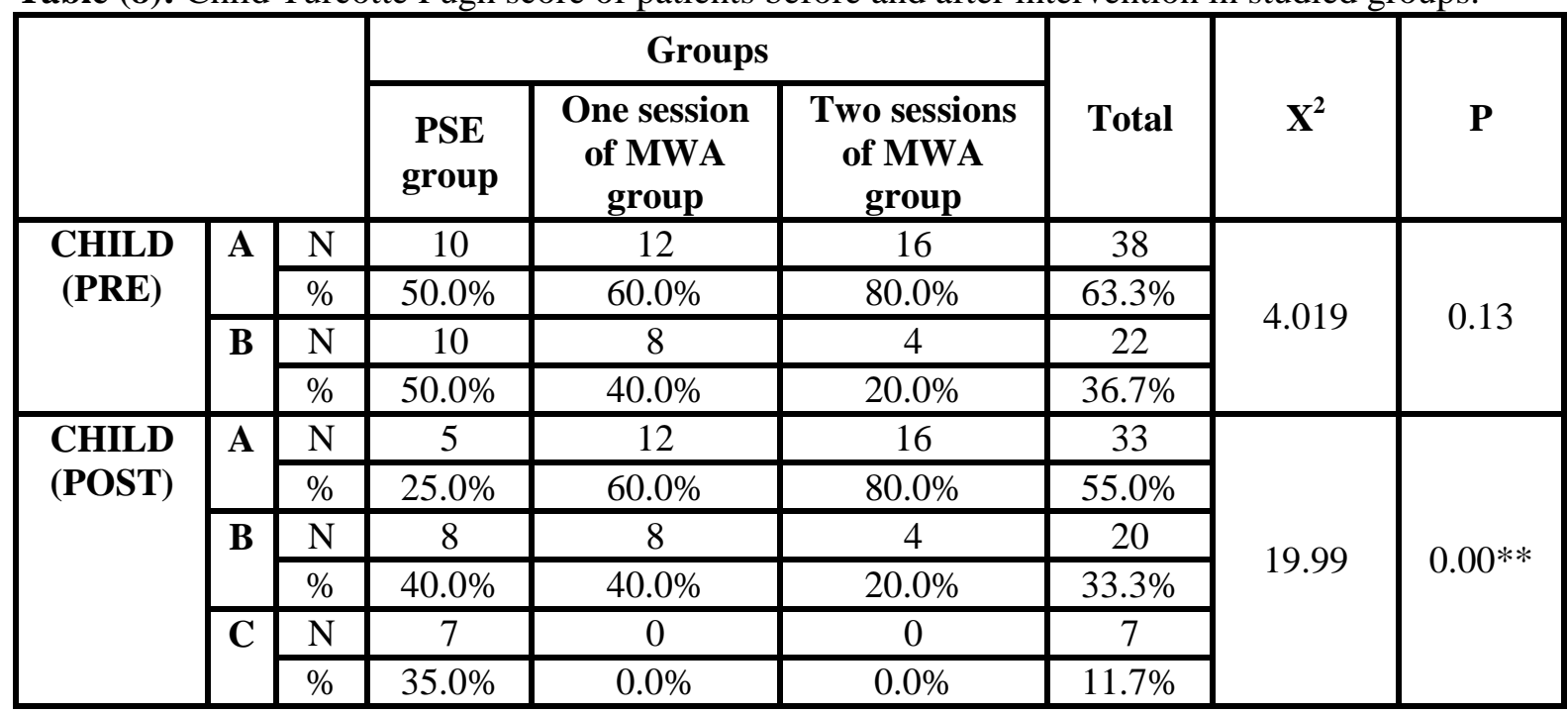

There is deterioration in child Turcotte Pugh in PSE group after 3 months $(\mathrm{P}=0.00)$ while no changes in other two groups, child class A was 10 patients and child class B was 10 patients and become child class A 5 patients, child class B 8 patients and child class C 7 patients. (PSE: partial splenic embolization, MWA: microwave ablation, X2: Chi-square). 
Table (9): Prevalence of complications among studied groups.

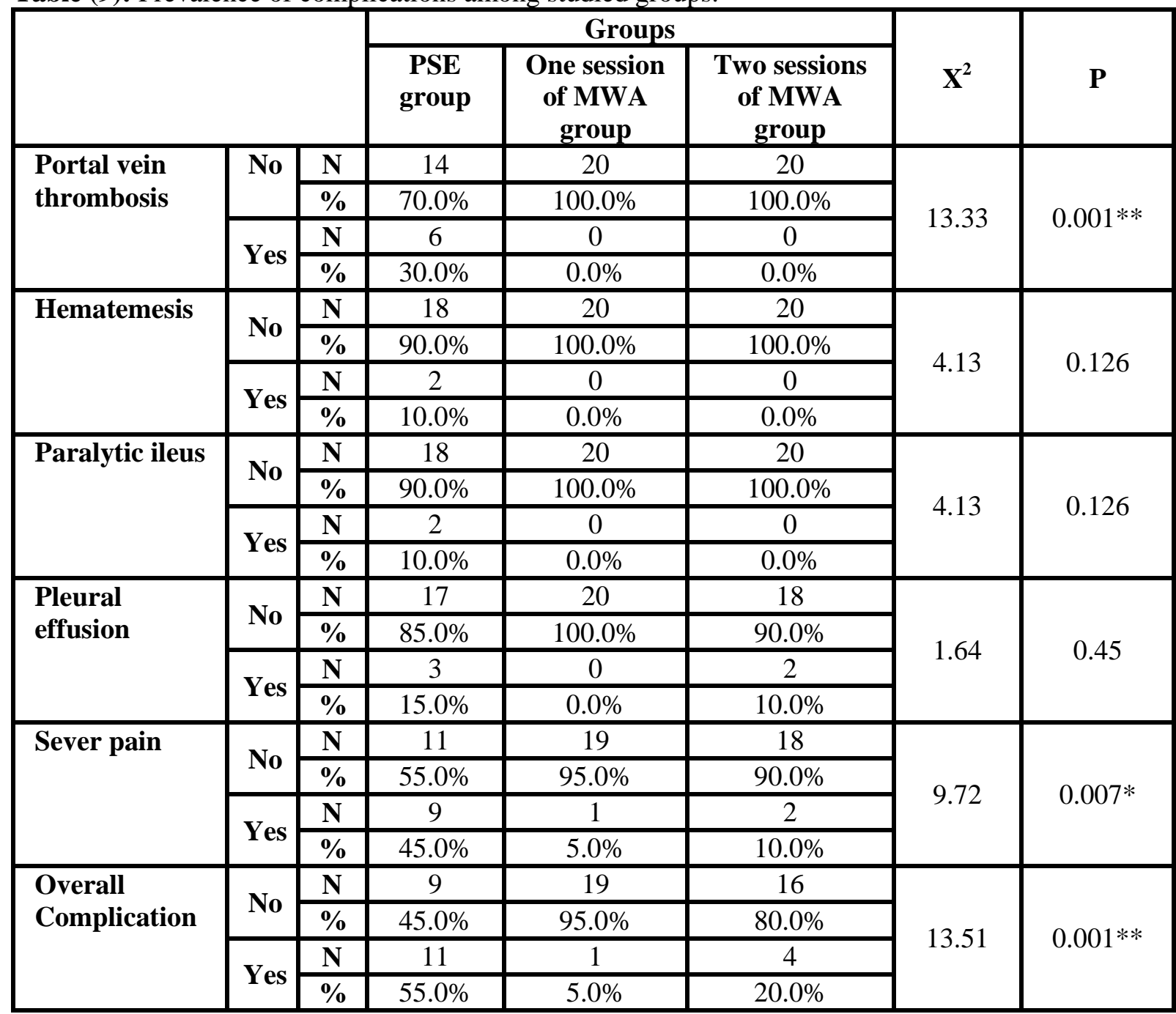

PSE group was associated with more serious complications than other two groups after 3 months. (PSE: partial splenic embolization, MWA: microwave ablation, X2: Chi-square, *: significant results, **: high significant results) 
Table (10): Laboratory parameters before and after the intervention of each group.

\begin{tabular}{|c|c|c|c|c|c|}
\hline \multicolumn{2}{|c|}{ Groups } & \multirow{2}{*}{$\begin{array}{c}\text { Mean } \\
9.4700\end{array}$} & \multirow{2}{*}{$\begin{array}{c}\text { SD } \\
2.14233\end{array}$} & \multirow{3}{*}{$\begin{array}{c}\begin{array}{c}\text { Paired } \\
\text { t/Sign }\end{array} \\
2.459\end{array}$} & \multirow{3}{*}{$\frac{\mathbf{P}}{0.023^{*}}$} \\
\hline \multirow{14}{*}{ PSE group } & HB pre & & & & \\
\hline & HB_3 month & 10.8800 & 2.35229 & & \\
\hline & Platelets pre & 42.75 & 15.4 & \multirow{2}{*}{12.514} & \multirow{2}{*}{$0.00 * *$} \\
\hline & Platelets_3 month & 252.7500 & 60.69759 & & \\
\hline & WBCs pre & 2.6 & 0.5 & \multirow{2}{*}{7.957} & \multirow{2}{*}{$0.000^{*}$} \\
\hline & WBCs_3 month & 6.3621 & 1.28952 & & \\
\hline & Albumin pre & 3.2050 & .36052 & \multirow{2}{*}{7.031} & \multirow{2}{*}{$0.00 * *$} \\
\hline & Albumin_3 month & 2.9850 & .30826 & & \\
\hline & Bilirubin pre & 1.4550 & .47404 & \multirow{2}{*}{3.664} & \multirow{2}{*}{$0.002 *$} \\
\hline & Bilirubin_3 month & 4.5200 & 3.86980 & & \\
\hline & INR pre & 1.1850 & .20072 & \multirow{2}{*}{5.620} & \multirow{2}{*}{$0.00 * *$} \\
\hline & INR_3 month & 1.5450 & .26848 & & \\
\hline & Creatinine pre & 1.0950 & .21145 & \multirow{2}{*}{7.571} & \multirow{2}{*}{$0.00 * *$} \\
\hline & Creatinine_3month & 1.5200 & .26077 & & \\
\hline \multirow{14}{*}{$\begin{array}{l}\text { One session of } \\
\text { MWA group }\end{array}$} & HB pre & 9.3750 & 2.02351 & \multirow{2}{*}{3.956} & \multirow{2}{*}{$0.001 * *$} \\
\hline & HB_3 month & 11.7600 & 1.10711 & & \\
\hline & Platelets pre & 45.75 & 11.28 & \multirow{2}{*}{9.031} & \multirow{2}{*}{$0.00 * *$} \\
\hline & Platelets_3 month & 126.9500 & 27.82365 & & \\
\hline & WBCs pre & 2.8 & 0.58 & \multirow{2}{*}{4.916} & \multirow{2}{*}{$0.00 * *$} \\
\hline & WBCs_3 month & 5.1550 & 1.31408 & & \\
\hline & Albumin pre & 3.4250 & .51593 & 0408 & 0624 \\
\hline & Albumin_3 month & 3.4050 & .61342 & 0.498 & 0.024 \\
\hline & Bilirubin pre & 1.1850 & .49234 & 6020 & $000 * *$ \\
\hline & Bilirubin_3 month & 1.5150 & .65957 & 0.020 & \\
\hline & INR pre & 1.2300 & .21546 & 3621 & $0002 *$ \\
\hline & INR_3 month & 1.3000 & .22942 & $5.0<1$ & 0.002 \\
\hline & Creatinine pre & .9600 & .21374 & 3052 & $\cap 0 \cap 7 *$ \\
\hline & Creatinine_3 month & 1.0650 & .16631 & 5.053 & $0.00 /$ \\
\hline & HB pre & 8.2050 & 2.15687 & 7249 & $000 * *$ \\
\hline & HB_3 month & 12.350 & 1.29553 & 1.249 & $0.00^{n}$ \\
\hline & Platelets pre & 40.6 & 11.5 & 11522 & $000 * *$ \\
\hline & Platelets_3 month & 155.9500 & 26.43259 & $11 . J \angle 2$ & 0.00 \\
\hline & WBCs pre & 2.4 & 0.55 & 5870 & $\cap \cap \cap * *$ \\
\hline & WBCs_3 month & 6.1800 & 1.27229 & $5.0 / 0$ & 0.00 \\
\hline Two sessions & Albumin pre & 3.6050 & .52060 & & \\
\hline group & Albumin_3month & 3.6250 & .57205 & 0.566 & 0.585 \\
\hline & Bilirubin pre & 1.4100 & .53988 & 6795 & $000 * *$ \\
\hline & Bilirubin_3 month & 1.7850 & .54799 & 0.193 & 0.00 \\
\hline & INR pre & 1.2500 & .21643 & 2698 & $0014 *$ \\
\hline & INR_3 month & 1.3100 & .20494 & 2.090 & $0.014^{\circ}$ \\
\hline & Creatinine pre & .9400 & .20105 & & 0 \\
\hline & Creatinine_3 month & 1.4600 & 1.78426 & .252 & 20 \\
\hline
\end{tabular}

(HB: hemoglobin, WBCs: white blood cells, INR: international normalized ratio, PSE: partial splenic embolization, MWA: microwave ablation, *: significant results, **: high significant results) 


\section{DISCUSSION}

This prospective randomized study was conducted on 60 patients with hypersplenism due to liver cirrhosis.

In the present study, hemoglobin more increased in microwave ablation groups than PSE group and in the two sessions of microwave than one session. This is finding in accordance with Assal et al. [12], who found significant increase in hemoglobin levels in microwave group compared to PSE group after 3 months of follow up.

The present study showed that, Total leucocyte count and platelets starts to increased significantly from the $1^{\text {st }}$ week in the PSE and in the two sessions of microwave than one session. This is in accordance with Assal et al. [12], who found the same results after 1 month and with Liang et al. [11], who found that total leucocyte count and platelets starts to increase significantly in the two sessions of microwave than one session. Also, Lee et al. [13] and Kumar et al. [14], who found rapid improvement in leukocytes and platelets after PSE.

Partial splenic embolization increases platelet counts via two mechanisms: by (1) reducing the splenic size, thereby reducing the trapping of thrombocytes in the embolized spleen; and (2) decreasing levels of platelet-associated immunoglobulin $\mathrm{G}$ ( $\mathrm{IgG}$ ). Reduction of platelet-associated IgG leads to decreased immunologic induction of thrombocytopenia. The increase in leukocytes after PSE can be attributed to the activation of body defense mechanisms against infarcted splenic tissues and this is according to Firat et al [15], which can explain the significantly greater degree of increase of leukocytes after PSE than after MWA in our study due to large splenic tissue infarction.

The present study showed that there was significant deterioration in serum albumin and significant increase in bilirubin, INR, amount of ascites and child score after treatment in PSE than other both groups. This is in accordance with Assal et al. [12], Sakai et al. [16] and Hayashi et al. [17]. On the other hand, Liang et al. [11], found that only albumin increased but not significantly after microwave, however the other parameters showed no significant change.

In our study no deterioration in kidney functions after procedures due to good preparation before intervention and good hydration after intervention. This is in accordance with Liang et al. [11], who found that there were no significant changes in renal function. On the other hand, Assal et al. [12], found increased serum creatinine in partial splenic artery embolization group.

In our study, complications like pleural effusion, severe pain occurred more in PSE and the two sessions of microwave than one session. This is in agreement with Jiang et al. [18], who found less complication in microwave groups than PSE group and increased complications with the increased ablated areas with microwave ablation. Also, this was in agreement to other studies done by Cai et al. [19] and Gonsalves et al. [20], who found that tense ascites, bacterial peritonitis, and post-embolization syndrome (fever, pain, vomiting) were the complications after PSE. However, another study recorded no cases with portal vein thrombosis after PSE as reported by Hidaka et al. [21] but Assal et al. [12] reported five patients developed portal vein thrombosis after PSE due to rapid rise in platelets count which managed by anticoagulant. Also; another study recorded no cases with pleural effusion after PSE as reported by Gerlock et al. [22].

In our study there is no thermal injury of surrounding viscera. This has been correlated with a study done by Jiang et al. [18]. However, Yu et al. [23] reported potential thermal injury of surrounding viscera.

There was no post-operative mortality in all groups. This was in accordance with Liang et al. [11] and Jiang et al. [18].

However, Sakai et al. [16], reported one death also, N'Kontchou et al. [24], reported two deaths due to abscess formation and septic shock.

However, both procedures have same therapeutic principles, but MWA was safer than PSE as the latter caused more deterioration of liver functions, worsen degree of ascites, and deterioration of Child score. So, serious adverse events after PSE will make us to prefer MWA techniques to increase the efficacy without losing the safety and two sessions of MWA than one session of MWA.

\section{Funding: None.}

\section{Conflicts of interest: None.}

Limitations of the study: Small sample size, exclusion of child $\mathrm{C}$ patients and other causes of cytopenia. 


\section{CONCLUSION}

The most frequent complication associated with MWA was mild pain that responded to analgesic.

So, percutaneous MWA is safe procedure for treatment of hypersplenism as a consequence of cirrhosis of liver and portal hypertension with good results in two sessions of MWA group.

\section{REFERENCES}

1. Bowdler AJ: The clinical significance of the spleen. The Complete Spleen. Totowa, NJ: Humana Press, 2002; 139-155.

2. Dacie JV, Brain MC, Harrison CV, Lewis SM and Worlledge SM. Non-tropical idiopathic splenomeglay (primary hypersplenism). A review of 10 cases and their relationship to malignant lymphomas. Br J Haematol. 1969;17(4):317-33.

3. Ellis LD and Dameshek HI. The dilemma of hypersplenism. Surg. Clinic. Nor. Am.1975: 55: 227-285.

4. Soper NJ and Rikkers LF. Effect of operations for variceal hemorrhage on hypersplenism. Am J Surg 1982; 144:700- 703.

5. Lv Y, Lau WY, Li Y, Deng J and Han XM. Hypersplenism: history and current status. Experimental and Therapeutic Medicine, 2016, 12.4: $2377-2382$

6. Lemair J, Rosière A, Bertrand C, Bihin B, Donckier JE, Michel L, et al. Surgery for massive splenomegaly. BJS open, 2017, 1.1: 11-17.

7. Kojouri K, Vesely SK, Terrell DR and George JN. Splenectomy for adult patients with idiopathic thrombocytopenic purpura: a systematic review to assess long-term platelet count responses, prediction of response, and surgical complications. Blood 2004; 104: 2623-2634.

8. Maddison FE. Embolic therapy of hypersplenism. Invest Radiol 1973; 8: 280-281.

9. Sangro B, Bilbao I and Herrero I. Partial splenic embolization for the treatment of hypersplenism in cirrhosis. Hepatology 1993; 18: 309-314.

10. Matsuoka T, Yamamoto A and Okuma T. CTguided percutaneous radiofrequency ablation of spleen: a preliminary study. AJR 2007; 188:10441046.

11. Liang P, Gao Y, Zhang H. Microwave Ablation in the Spleen for Treatment of Secondary Hypersplenism: A Preliminary Study. AJR 2011; 196:692-696.

12. Assal F, El Kassas M, Esmail E, Elbadry AA, Abousaif S, Mahdy R, et al. Microwave ablation in the spleen versus partial splenic artery embolization: A new technique for hypersplenism in cirrhosis. Arab Journal of Gastroenterology. 2017 Mar 1;18(1):25-9.
13. Lee CM, Leung TK and Wang HJ. Evaluation of the effect of partial splenic embolization on platelet values for liver cirrhosis patients with thrombocytopenia. World J Gastroenterol.2007; 13(4):619-22.

14. Kumar A, Yoon J, Thakur V and Contractor S. Safety and efficacy of partial splenic embolization for hypersplenism: a meta-analysis. JVIR. 2013; 24(4) S138.

15. Firat A, Boyvat F, Moray G, Aytekin C, Karakayali $\mathrm{H}$ and Haberal M. Comparison of two different percutaneous splenic artery interventions in the treatment of hypersplenism: preliminary report. Transplant Proc.2005; 37:1094.

16. Sakai T, Shiraki K and Inoue H. Complications of partial splenic embolization in cirrhotic patients. Dig Dis Sci.2002; 47(2):388-91.

17. Hayashi H, Beppu T and Okabe K. Risk factors for complications after partial splenic embolization for liver cirrhosis. Br J Surg 2008; 95: 744-750.

18. Jiang XW, Gao $\mathrm{F}$ and Ma Y. Percutaneous microwave ablation in the spleen for treatment of hypersplenism in cirrhosis patients. Dig Dis Sci, 2015; published with open access at Springerlink.com accessed on 22 August 2015.

19. Cai M, Zhu K and Huang W. Portal Vein Thrombosis after Partial Splenic Embolization in Liver Cirrhosis: Efficacy of Anticoagulation and Long-term Follow up.J Vasc Interv Radiol. 2013; 24(12):1808-16.

20. Gonsalves CF, Mitchell EP and Brown DB. Management of hypersplenism by partial splenic embolization with ethylene vinyl alcohol copolymer. AJR Am J Roentgenol.2010; 195(5): 1241-4.

21. Hidaka H, Kokubu S, Nakazawa T, Minamino T, Takada J, Tanaka Y, et al. Therapeutic benefits of partial splenic embolization for thrombocytopenia in hepatocellular carcinoma patients treated with radio frequency ablation. Hepatol Res.2009; 39(8):772-8.

22. Gerlock AJ, MacDonnell RC and Muhletaler CA. Partial splenic embolization for hypersplenism in renal transplantation. AJR Am J Roentgenol. 1982; 138:451.

23. Yu J, Liang P, Yu XL, Cheng ZG, Han ZY, Mu MJ, et al. US-guided percutaneous microwave ablation of renal cell carcinoma: Intermediate term results. Radiology. 2012; 263: 900-908.

24. N'Kontchou G, Seror O and Bourcier V. Partial splenic embolization in patients with cirrhosis: efficacy, tolerance and long-term outcome in 32 patients. Eur J Gastroenterol Hepatol 2005; 17: 179-184. 